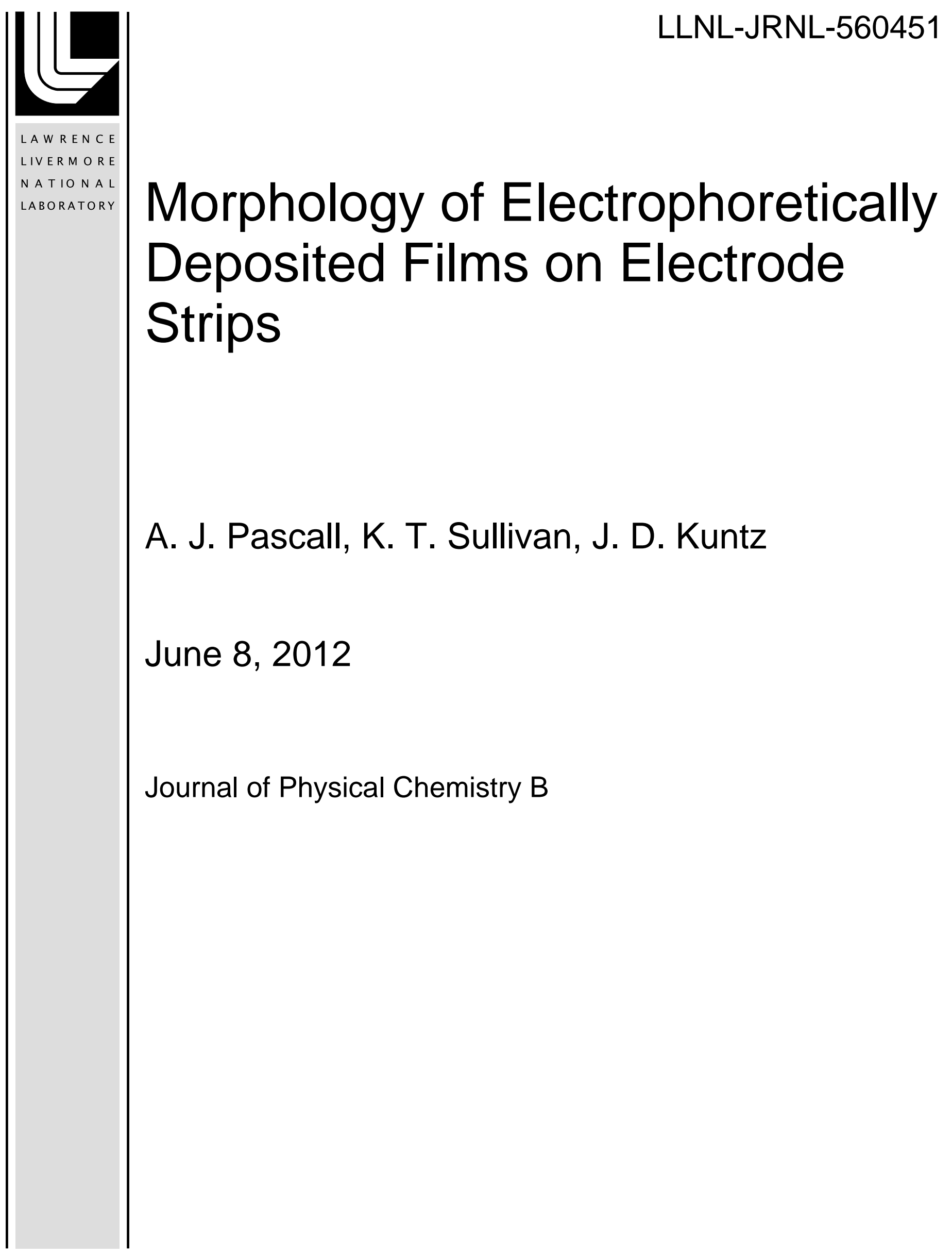


This document was prepared as an account of work sponsored by an agency of the United States government. Neither the United States government nor Lawrence Livermore National Security, LLC, nor any of their employees makes any warranty, expressed or implied, or assumes any legal liability or responsibility for the accuracy, completeness, or usefulness of any information, apparatus, product, or process disclosed, or represents that its use would not infringe privately owned rights. Reference herein to any specific commercial product, process, or service by trade name, trademark, manufacturer, or otherwise does not necessarily constitute or imply its endorsement, recommendation, or favoring by the United States government or Lawrence Livermore National Security, LLC. The views and opinions of authors expressed herein do not necessarily state or reflect those of the United States government or Lawrence Livermore National Security, LLC, and shall not be used for advertising or product endorsement purposes. 


\title{
Morphology of Electrophoretically Deposited Films on Electrode Strips
}

\author{
Andrew J. Pascall, * Kyle T. Sullivan, and Joshua D. Kuntz \\ Lawrence Livermore National Laboratory, \\ 7000 East Avenue, Livermore, California 94550, USA \\ E-mail: pascall1@IInl.gov
}

*To whom correspondence should be addressed 


\begin{abstract}
Studies of the kinetics of electrophoretic deposition (EPD) processes have generally focused on electrode geometries that yield analytical solutions, such as infinite parallel planes and concentric cylinders. In this article, we construct a finite element model for EPD of material onto a planar strip electrode which shows excellent qualitative agreement to experimental results in a similar system. Notably, we demonstrate that the presence of the edges of the electrode lead to a singularity in the electric field that significantly effects the morphology of the deposit at short times or for thin deposits.
\end{abstract}

Keywords: EPD, finite element analysis, edge effects, colloidal deposition, directed assembly, numerical modeling

\title{
Introduction
}

Electrophoretic deposition (EPD) is an industrially relevant process ${ }^{1}$ in which colloidal particles suspended in a liquid are forced to deposit onto an electrode under an applied electric field. While EPD was originally developed as a process for painting metals, ${ }^{2,3}$ it is now recognized as a general method for depositing material on any conductive surfaces and has found diverse applications in areas such as thermal barrier coatings, ${ }^{4,5}$ transparent ceramics, ${ }^{6,7}$ solar cells,,${ }^{8,9}$ fuel cells, ${ }^{10,11}$ solid state lighting and displays, ${ }^{12,13}$ and medical implants. ${ }^{14-18}$

The first theoretical treatment of the deposition kinetics of EPD was performed by Hamaker ${ }^{19}$ who predicted that the mass of the deposited film on an unpatterned planar electrode is proportional to the charge passed at the electrodes. Later, Avgustinik, et al. ${ }^{20}$ extended Hamaker's theory to depositions on concentric cylindrical electrodes. Hamaker's original analysis assumed that the concentration of suspended solids is constant. Sarkar and Nicholson ${ }^{21}$ review and extend theory that relaxes the constant concentration assumption and provide a general expression for the kinetics that is valid for infinite planar geometries under constant current and constant voltage conditions. Biesheuvel and Verweij ${ }^{22}$ derive the Hamaker-Avgustinik theory from a general theory of 
sedimentation proposed by Kynch, ${ }^{23}$ while González-Cuenca, et al. ${ }^{24}$ show that smooth deposits can only form when the deposit permittivity is lower than the suspension permittivity. Ferrari and Moreno $^{25}$ provide a comprehensive review of EPD kinetics studies.

While it is evident that considerable work has been done to model the EPD process analytically under various conditions in planar and cylindrical geometries, more complicated geometries have not generally yielded analytical solutions, but rather have been studied numerically. ${ }^{26,27}$ One such experimentally relevant geometry, the planar strip electrode, ${ }^{13,28-30}$ seems to have escaped the attention of the modeling community. A key feature of this geometry, which is absent in the geometries studied previously, is the presence of electrode edges which we show locally enhance the electric field and significantly affect the morphology of the deposit. In this article, we use finite element analysis to develop a model of the electrophoretic deposition of material onto a metallic strip under potentiostatic conditions in the geometry studied experimentally by Sullivan, et al. ${ }^{28,29}$ - a finite width strip electrode opposing a parallel planar electrode. We also provide an experimental study of such a deposition and show that the model qualitatively captures the key trends.

\section{Modeling Electrophoretic Deposition onto an Electrode Strip}

Let us begin by considering an electrode of width $2 W$ embedded in a insulating substrate separated from a counter electrode by a distance $H$ (Figure 1a). A suspension of permittivity $\varepsilon$ and viscosity $\eta$ containing particles at a concentration $c_{0}$ is placed between the electrodes. A potential of $V_{a p p}$ is applied to the counter electrode while the deposition electrode is grounded. Under the action of the applied electric field, the particles migrate through the suspension and accumulate at the deposition electrode forming a deposit. The goal of this work is to model this deposition process and determine the shape of the deposit as a function of time.

The electrophoretic deposition of particles onto the electrode strip was modeled by formulating the particle transport equations and solving for the time-dependent concentration profile in a $2 \mathrm{D}$ 

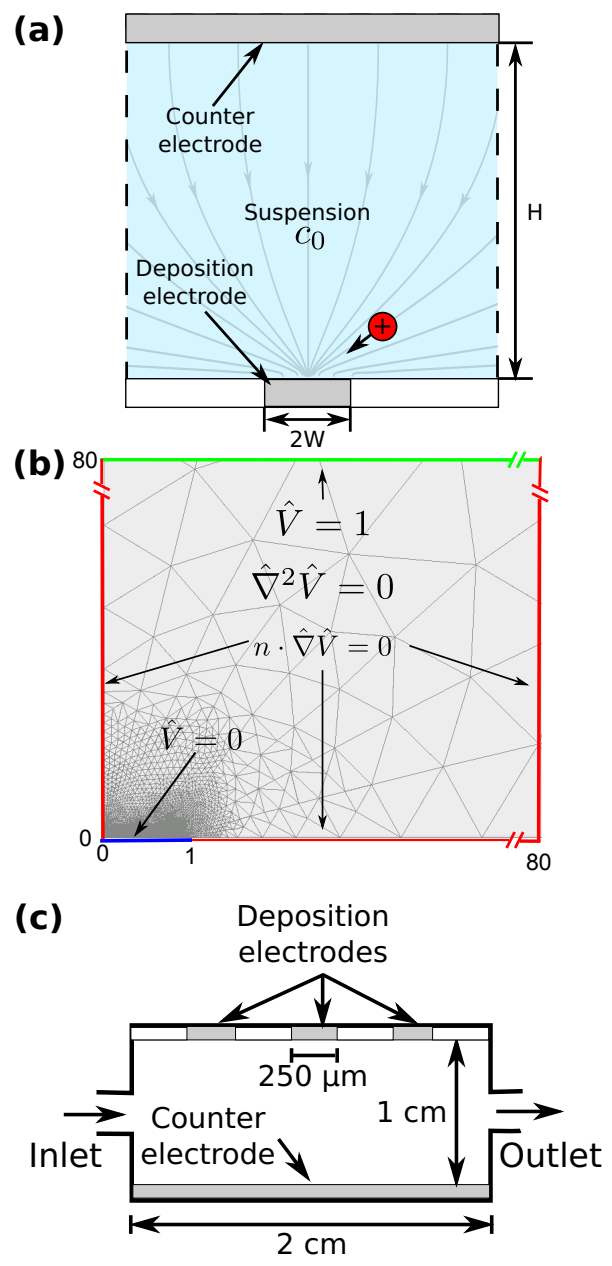

Figure 1: (a) Schematic of the model system. The deposition electrode of extent $2 \mathrm{~W}$ is separated from a counter electrode by a distance $H$. The space between the electrode is filled with a suspension containing particles at concentration $c_{0}$. The charged nanoparticle (red) follows electric field lines and deposits onto the deposition electrode. (b) Due to the symmetry of the model system about the center line of the deposition electrode, the computational domain represents half of model system with the origin fixed at the deposition electrode center and a line of symmetry at $\hat{x}=0$. The dimension are scaled to the electrode half width and represent those of the experimental system. The mesh for the finite element solution was constructed such that the mesh density is increased in the vicinity of the deposition electrode with the highest density near the edge of the electrode. The electrical boundary conditions are: $\hat{V}=0$ (blue); $\hat{V}=1$ (green); no current, $d \hat{V} / d n=0$ (red). (c) Schematic of the experimental system (not to scale). A trio of deposition electrodes $(250 \mu \mathrm{m}$ wide, separated by $6 \mathrm{~mm}$ center-to-center) face a planar counter electrode across distance of $1 \mathrm{~cm}$. The suspension is loaded into the cell through the inlet with the cell rotated vertically. During deposition and subsequent suspension withdrawal, the cell is rotated $90^{\circ}$ such that the deposition electrode is on top of the counter electrode, as depicted. 
domain (Figure 1b) under the following assumptions: The suspension is a fixed volume; no fluid flows into or out of the domain. The suspension is electroneutral at all points and the particles move through a quiescent fluid. The electric field is not perturbed by the presence of the particles or the deposit (equivalent to assuming that the deposit and suspension have equivalent permittivities or conductivities), nor are capacitive or Faradaic currents at the electrode considered. The particles move through the fluid via interaction with an applied electric field and by diffusion; however, particle-particle interactions are neglected except for an excluded volume effect that limits maximum packing and manifests as a volume fraction dependent viscosity. Higher order electrokinetic effects such as dielectrophoresis ${ }^{31}$ and induced charge electrophoresis ${ }^{32}$ are not treated. Finally, all particles are treated as identical and the gravitational forces on the particles are neglected.

Given that the suspension is electroneutral and that the presence of the deposit does not affect the electric field, the electrostatic potential in the domain is governed by Laplace's equation:

$$
\nabla^{2} V=0
$$

where $\mathrm{V}$ is the electrostatic potential at a point in the domain and the electric field is

$$
E=-\nabla V
$$

The electric field is coupled to the total flux of particles in the domain,

$$
j=-D \nabla c+\mu E c
$$

where $c$ is the concentration of particles, $D$ and $\mu$ are their diffusivity and electrophoretic mobility, respectively. The first term on the RHS of (3) represents the diffusion of particles in the suspension, while the second term represents electrophoresis of the particles. The number of particles in the 
domain is conserved; thus, the concentration evolves with respect to time according to:

$$
\begin{aligned}
\frac{d \phi}{d t} & =-\nabla \cdot j \\
& =\nabla \cdot(D \nabla \phi+\phi \mu \nabla V),
\end{aligned}
$$

where we have written the concentration more conveniently in terms of the volume fraction, $\phi=$ $V_{p} c$, where $V_{p}$ is the volume occupied per particle. Additionally, (2) has been used to show explicit coupling of the concentration field to the electrostatics. While (4) resembles the Nernst-Planck equation for ion transport in electrolytes, the mobility of the particles here is the electrophoretic mobility and is not given by the Stokes-Einstein relation. The electrophoretic particle mobility is determined by solving for the electrokinetic flow around the particle, the details of which are presented elsewhere. ${ }^{33}$ While a general relation for the mobility was provided by Henry, we will restrict ourselves to the thick double layer regime of Hückel $^{34}$ for simplicity and to more closely match the experimental conditions:

$$
\mu=\frac{2 \varepsilon \zeta}{3 \eta},
$$

where $\zeta$ is the zeta potential of the particles and $\eta$ the suspension viscosity.

Since each particle occupies a finite volume in the suspension, there is a maximum volume fraction, $\phi_{\max }=\phi_{r c p}=0.64$ assuming a randomly closed packed deposit, ${ }^{35}$ which cannot be exceeded. This constraint is enforced by assuming a Mooney-type ${ }^{36}$ dependence of viscosity on volume fraction:

$$
\frac{\eta}{\eta_{0}}=\exp \left(\frac{2.5 \phi}{1-\phi / \phi_{\max }}\right),
$$

where $\eta_{0}$ is the pure solvent viscosity. Thus,

$$
\mu \rightarrow 0 \text { as } \phi \rightarrow \phi_{\max }
$$

In order to allow for the comparison of scales between terms in governing equations (1) \& (4) and for ease of solution, the problem was non-dimensionalized by choosing characteristic scales 
such that:

$$
\begin{aligned}
\hat{V} & =V / V_{a p p}, \\
\hat{\phi} & =\phi / \phi_{\text {max }}, \\
\hat{\nabla} & =W \nabla, \\
\hat{t} & =t / t_{c},
\end{aligned}
$$

where $t_{c}$ is a characteristic time scale, of which two emerge from non-dimensionalization: $t_{c}=$ $W^{2} / D$ and $t_{c}=W^{2} / \mu V_{a p p}$. The former is the characteristic time scale for a particle to diffuse across the electrode, while the latter represents the characteristic time of a particle to convect a distance $W$ by electrophoresis. Since electrophoretic motion dominates in the experimental system, the latter time scale is chosen and $t_{c}=2.0$ seconds for the experimental conditions. This leads to the following set of governing equations for the system:

$$
\begin{aligned}
\hat{\nabla}^{2} \hat{V} & =0 \\
\frac{d \hat{\phi}}{d \hat{t}} & =\hat{\nabla} \cdot\left[\mathrm{Pe}^{-1} \hat{\nabla} \hat{\phi}+\exp \left(-2.5 \phi_{\max } \frac{\hat{\phi}}{1-\hat{\phi}}\right) \hat{\phi} \hat{\nabla} V\right]
\end{aligned}
$$

where $\mathrm{Pe}=\frac{2 \pi \varepsilon \zeta V_{a p p} d}{k_{B} T}$ is the Péclet number and the diffusivity of the particle has been related to particle diameter, $d$, via the Stokes-Einstein relation:

$$
D=\frac{k_{B} T}{3 \pi \eta_{0} d}
$$

Note that the viscosity employed in Stokes-Einstein relation is the pure solvent viscosity, $\eta_{0}$, and is not volume fraction dependent. This choice is necessary to maintain numerical stability in the finite element solver. While undoubtedly the diffusivity of a particle will decrease at high volume fractions, the error in assuming that the diffusivity is volume fraction independent will be most pronounced at low values of the Péclet number where diffusion is most important. For the simulations presented here $\mathrm{Pe}=1000$, so this assumption is justified. 


\section{Performing the simulations}

Due to the symmetry of the system, the computational domain consisted of half of the model domain with the computation origin placed at the center of the deposition electrode (Figure 1b). The aspect ratio of the experimental cell was preserved resulting in a domain that is $80 \mathrm{x} 80$. In this domain, the boundary conditions on the governing equations are given by: $\hat{V}=0$ at the deposition electrode surface, $\hat{V}=1$ at the counter electrode surface, and $d \hat{V} / d n$ at other surfaces and the plane of symmetry, where $n$ is the direction normal to the surface. The boundary condition on the concentration is no flux of material at all boundaries and at the plane of symmetry. While the initial condition is that $\hat{\phi}=\hat{\phi}_{0}$ at all points in the domain.

The governing equations (8) \& (9) were solved using commercial finite element software (COMsOL, v4.2). The initial mesh was constructed of $\sim 61000$ triangular elements such that the mesh density is increased in the vicinity of the deposition electrode with the highest density near the edge of the electrode in order to capture the singularity in the electric field that occurs there. Adaptive mesh refinement was utilized over the course of the simulation to increase mesh density near large gradients in volume fraction. The solution to (8) was found using the built-in electrostatics module, while (9) was implemented as a generic PDE. Time stepping was performed using the BDF method with a MUMPS solver.

Stable solutions to the governing equations were found up to $\mathrm{Pe}=1000$ for the experimental initial condition $\hat{\phi}_{0}=3.125 \times 10^{-3}$. The solution did not converge for Pe $>1000$. Thus, it was not possible to quantitatively simulate the experiments which were performed at $\mathrm{Pe} \sim 64000$; however, qualitative comparisons can be made.

The simulations were performed with a temporal resolution of $\Delta \hat{t}=1$ for $\hat{t}$ between 0 and 1000 . By $\hat{t}=1000$, the total depletion of the suspension was $\sim 5 \%$ which approximately corresponds to that of the experiments. The simulations were continued at a lower temporal resolution of $\Delta \hat{t}=10$ for $\hat{t}$ between 1000 and 5000 in order to observe the long term evolution of the deposit. 


\section{Experimental Methods}

\section{Suspension Preparation}

A suspension containing $0.063 \mathrm{vol} \% \mathrm{CuO}$ nanoparticles (Sigma-Aldrich, aggregates approximately $100 \mathrm{~nm}$ in diameter) and $0.137 \mathrm{vol} \% \mathrm{Al}$ nanoparticles (Novacentrix, $80 \mathrm{~nm}$ ) for a total $0.2 \mathrm{vol} \%$ solids was suspended in a 3:1 by volume ethanol:deionized water mixture by first suspending the particles in absolute ethanol (Pharmco-Aaper) and sonicating with an ultrasonic wand for 1 minute (50\% duty cycle). The DI water (Milli-Q, Millipore) was then added to the suspension and it was again sonicated with an ultrasonic wand for 1 minute (50\% duty cycle). The zeta potential of the suspended particles was measured with a ZetaSizer Nano ZS90 (Malvern) and yielded a single broad peak at $32 \pm 14 \mathrm{mV}$.

\section{Electrophoretic Deposition}

Depositions were carried out in a custom built flow chamber that consists of a chamber of dimensions $2 \times 2 \times 1 \mathrm{~cm}$ with 1/16" ID inlet and outlet ports (Figure 1c). The cell is assembled such that the deposition electrode and counter electrode are separated by $1 \mathrm{~cm}$. The deposition electrode consists of a trio of $250 \mu \mathrm{m}$ wide photolithographically patterned Pt strips separated by $6 \mathrm{~mm}$ center-to-center on a glass microscope slide and $20 \mathrm{~mm}$ in length (L), while the counter electrode is a $2 \times 2 \mathrm{~cm}$ unpatterned silicon wafer sputtered with a Pt-Cr thin film. The flow chamber was filled in a vertical orientation at $10 \mathrm{~mL} / \mathrm{min}$ with the freshly made suspension using a syringe pump (PHD ultra, Harvard Apparatus) connected to the inlet. The flow was continued until the suspension was seen exiting through the outlet, indicating that the chamber was full. The flow chamber was then rotated such that the deposition electrode was above the counter electrode (Figure 1c).

The depositions were carried out with the electrodes oriented horizontally under potentiostatic conditions by applying a constant $40 \mathrm{~V}_{\mathrm{DC}}$ across the electrodes for a specified amount of time ranging from 5 to 180 seconds $(\hat{t}=2.5$ to 90$)$. After the deposition was complete, the electric field was turned off and the remaining suspension was withdrawn at $2 \mathrm{~mL} / \mathrm{min}$ through the inlet 
port to prevent disturbing the deposited film. The chamber was then disassembled and the EPD film was allowed to dry under ambient conditions. The cross-sectional profile of the deposits was determined by laser profilometry (Optimet ConoScan 3000, Optical Metrology, Ltd.) (Figure 3b).

\section{Results and Discussion}
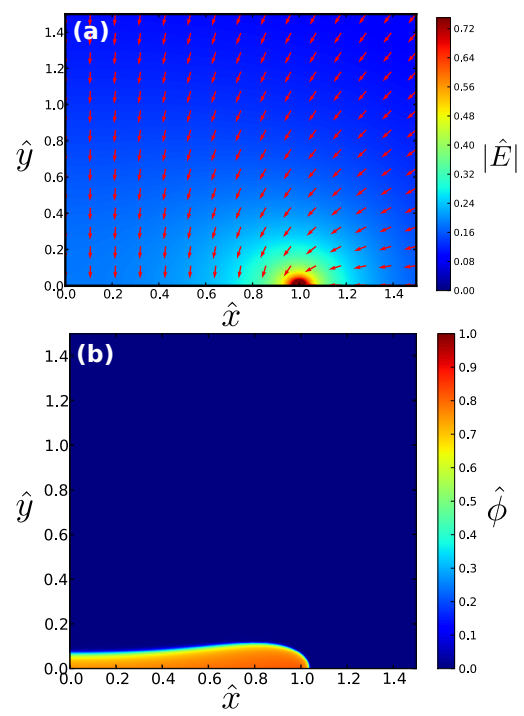

Figure 2: (a) The electric field in the vicinity of the deposition electrode. The color represents the magnitude of the electric field, $|\hat{E}|$, while the arrows indicate the direction. A singularity in the electric field at the edge of the electrode $(\hat{x}=1)$ is clearly evident. (b) The concentration field of particles at $\hat{t}=75$ for $\mathrm{Pe}=1000$. Note, that the presence of the electric field singularity leads to a high particle flux near the edge of the electrode leading to a deposit that is thicker near the edge than in the center at short times.

As evident in Figure 2a, a singularity in the electric field occurs at the edge of the electrode. This is a general feature found any place where there is a spatial discontinuity in the electrical boundary conditions, such as at the edge of the electrode where the boundary condition goes from $\hat{\phi}=0$ to $d \hat{\phi} / d n=0$. The singularity has a profound effect on the structure of the deposit, particularly at short times. Since the flux of particles is proportional to the electric field (3), a high flux of particles initially occurs in the vicinity of the edge. This causes the film to grow the fastest near the edge which results the maximum height of the deposit occurring near the edge ("wings"). This behavior is captured in both the simulated deposits (Figure 3a) as well as the experimental 

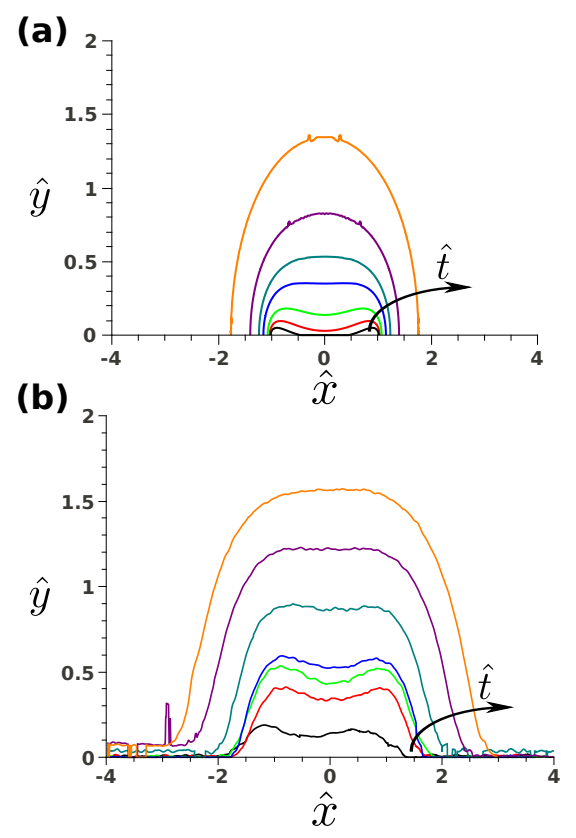

Figure 3: (a) The cross-sectional profile of the deposit from simulations for $\mathrm{Pe}=1000$ for $\hat{t}=$ 50 (black), 100 (red), 200 (green), 400 (blue), 600 (cyan), 1000 (purple), 2000 (orange). The surface of the deposit was chosen to coincide with the $\hat{\phi}=0.75$ contour. Numerical artifacts in the solution are evident as ripples on the simulated deposit surface at large times and are caused by the decreased mesh density in that area. Note that the simulations results have been reflected across $\hat{x}=0$ in order to more closely resemble the experimental cross-sections. (b) The mean experimental cross-sectional profile of the deposit at $\mathrm{Pe}=64000$ determined by averaging the profile at 5 locations along the strip length for $\hat{t}=2.5$ (black), 10 (red), 15 (green), 20 (blue), 36.5 (cyan), 60 (purple), 90 (orange) as determined by laser profilometry. 
(Figure 3b) (Note that for this analysis, we have defined the $\hat{\phi}=0.75$ contour as the surface of the simulated deposit). At longer times, the growing film front advances away from the singularity, the particle flux at the electrode edge decreases compared to the flux at the electrode center line, and the position of the maximum height shifts from the edge of the electrode to the center line. As noted by Deconinck, ${ }^{37}$ similar behavior is seen in electroplating processes near electrode edges, except since the free surface of the deposit is conductive, the wings continue to grow. Figure 4a shows the position of the maximal height of the deposit along $\hat{x}$ axis which reflects this behavior. It also reveals excellent qualitative agreement between the simulated and experimental evolution of the shape of the deposit. Far from the deposition electrode, the electric field approximates the field between a plate and a point charge, and the deposit begins to grow isotropically into a semicircular shape. A video of the deposition process up to $\hat{t}=1000$ is provided in the supplemental information.

In addition to the film growing vertically from the deposition electrode, it also grows laterally away from the electrode edge and covers part of the insulating region adjacent to the electrode. The reason for this lateral growth is apparent from the electric field shown in Figure 2a: The boundary condition on the insulating surface requires that there be only a tangential component to the electric field near the surface. Since the flux of particles follows electric field lines, particles that reside close to the electrode surface are forced horizontally along the insulating surface until they encounter the growing film front. Figure $4 \mathrm{~b}$ plots the aspect ratio of the film, here defined as the height of the deposit at the electrode centerline to the half of the lateral extent. After some induction period, during which time the deposit does not cover the entire electrode, the aspect ratio initially increases rapidly as the deposit grows in height faster than it grows in width. At longer times, the aspect ratio tends to level off representing an isotropic growth mode. Again, there is excellent qualitative agreement between simulations and the experimentally determined aspect ratio.

The dimensionless mass of the deposited film per unit length as a function of time can be 

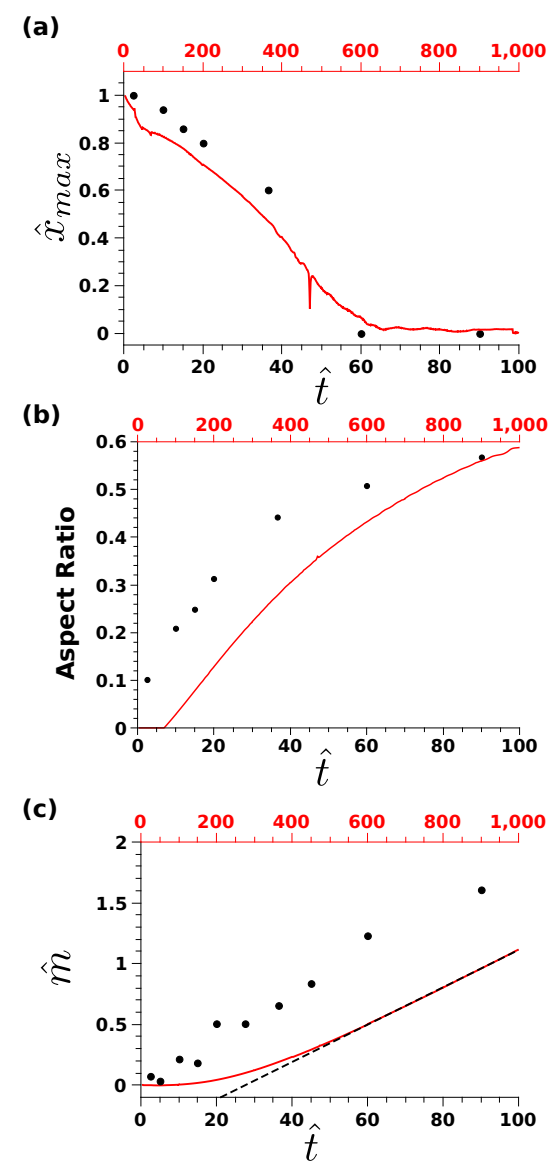

Figure 4: (a) The $\hat{x}$ position of the maximum thickness of the deposit $\left(\hat{x}_{\text {max }}\right)$ versus deposition time. Initially, the deposit is thickest at the electrode edge $(\hat{x}=1)$ due to the electric field singularity occurring there. At later times, the thickest part of the deposit moves toward the electrode centerline $(\hat{x}=0)$ since, away from the singularity, the highest particle flux occurs along the centerline. (b) The deposit aspect ratio as defined as the ratio of the maximum height of the deposit to half of the maximum width versus deposition time. Initially, the deposit grows faster vertically than horizontally, but after some time, the aspect ratio approaches a constant value reflecting isotropic growth. (c) The scaled mass per unit length $(\hat{m})$ of the deposit versus deposition time, which is calculated from the simulations as the integrated volume fraction between the $\hat{\phi}=0.75$ and the electrode surface given by (10). The dashed line represents a linear fit to the simulation data for $\hat{t}=600-1000$. Experimental points $(\bullet)$ follow the lower axis while simulated depositions (solid line) follow the upper. 
extracted from the model by integrating the volume fraction contained within the film:

$$
\hat{m}=\int \hat{\phi} d \hat{A}
$$

This is plotted in Figure 4c along with the experimentally determined scaled mass per unit length. The experimental mass per unit length was determined by dividing the mass of the deposited film by the characteristic mass scale, $m_{c}=n \rho \phi_{\max } W^{2} L$, where $n=3$ is the number of identical strips per substrate and $\rho=4.6 \mathrm{~g} / \mathrm{cm}^{3}$ is the density of a fully dense deposit as calculated from elemental composition via ICP-OES. ${ }^{28}$ At longer times, the simulation curve is well fit by $\hat{m}=$ $-0.425+0.00153 \hat{t}\left(R^{2}=0.999\right.$, dashed line in Figure $\left.4 \mathrm{c}\right)$. The linear dependence of mass on time can be justified because at long times, the electric field at the surface of the deposit is approximated by the field around a point charge located at the origin, and thus the flux of material, which is proportional to the electric field, goes like $r(t)^{-1}$, while the surface area of the deposit goes like $r(t)$, where $r(t)$ is the distance from the center of the electrode to the film surface.

It should be noted that there are three growth regimes; however, only the first two are captured in these simulations. At early times, which occurs when $r(t) \ll W$, deposit growth is dominated by the electric field singularity at the edges of the electrode, while at intermediate times when $W \lesssim r(t) \ll H$, isotropic growth occurs because the structure of the electric field at the deposit surface approaches that of a point charge at the origin. When $r(t) \sim H$, a regime that is not explored in this work, the electric field near the deposit surface appears like that of a dipole with one pole at the center of the electrode and the opposing pole at $2 \mathrm{H}$.

\section{Conclusions}

In this work, we have shown that the morphology of an electrophoretically deposited film on a strip electrode is dominated at short times by the intense electric field at the electrode edges which results in a deposit with wings near the edges. These intense electric fields occur any time there is an abrupt change in electrostatic boundary conditions, such as occurs at the edge of an electrode. 
Thus, deposits of nonuniform thickness should be expected to occur in general in such cases. Over time, as the depositing front moves away from the electrode surface, the influence of the electric field singularity at the edge lessens, the deposit wings disappear, and the deposit tends to grow in a more isotropic manner.

While the simulation was unable to attain the correspondingly high Péclet number encountered in the experiment, the simulations at $\mathrm{Pe}=1000$ show excellent qualitative agreement with experiment. This provides confidence that relevant and reasonable physics are included in the simulation and allow us to conclude that a key feature of EPD on electrodes that have edges or corners will be a singularity of the electric field near the edge that will initially lead to a thicker deposit in that region. However, additional physics may also be required to correctly model deposition over a wider range of experimental conditions. Perhaps the most relevant physics that we have neglected here is to allow for a voltage drop across the deposit. Several authors, going back to Hamaker

himself, ${ }^{19,21,22,24}$ have invoked this to explain trends in deposited mass versus time. In the future, we hope to resolve the high Péclet number model convergence issues and demonstrate the ability to quantitatively predict the deposition kinetics for a wide range of experimental conditions.

\section{Acknowledgments}

A.J.P. would like to thank Todd Weisgraber for helpful discussions. This work was funded by the Laboratory Directed Research and Development Strategic Initiative program 11-SI-005 "Disruptive Fabrication Technologies Initiative" and performed under the auspices of the US Department of Energy by Lawrence Livermore National Laboratory under Contract DE-AC52-07NA27344. LLNL-JRNL-560451.

\section{Supporting Information}

A video of the simulation at $\mathrm{Pe}=1000$ for $\hat{t}=0-1000$. Mesh refinement occurs every 100 time steps which increases mesh density in regions with large gradients in concentration. This 
information is available free of charge via the Internet at http://pubs.acs.org.

\section{References}

(1) Clasen, R. Preparation of High-Purity Glasses and Advanced Ceramics Via EPD of Nanopowders. In Electrophoretic Deposition of Nanomaterials, 1st ed.; Nanostructure Science and Technology; 2012; pp 217-266.

(2) Davey, W. P. Process for Making and Applying Japan, 1919, United States Patent 1,294,627.

(3) Burnside, G. L.; Brewer, G. Electrophoretic Coating Process, 1965, United States Patent $3,200,057$.

(4) Lu, X.; Xiao, P. J. Eur. Ceram. Soc. 2007, 27, 2613-2621.

(5) Van der Biest, O.; Joos, E.; Vleugels, J.; Baufeld, B. J. Mater. Sci. 2006, 41, 8086-8092.

(6) Braun, A.; Falk, G.; Clasen, R. Materialwiss. Werkstofftech. 2006, 37, 293-297.

(7) Tabellion, J.; Zeiner, J.; Clasen, R. J. Mater. Sci. 2006, 41, 8173-8180.

(8) Salant, A.; Shalom, M.; Hod, I.; Faust, A.; Zaban, A.; Banin, U. ACS Nano 2010, 4, 59625968.

(9) Miyasaka, T.; Kijitori, Y. J. Electrochem. Soc. 2004, 151, A1767.

(10) Munakata, H.; Ishida, T.; Kanamura, K. J. Electrochem. Soc. 2007, 154, B1368.

(11) Morikawa, H.; Tsuihiji, N.; Mitsui, T.; Kanamura, K. J. Electrochem. Soc. 2004, 151, A1733.

(12) Talbot, J. Electrophoretic Deposition of Phosphors for Information Displays and Solid State Lighting. In Electrophoretic Deposition of Nanomaterials; Nanostructure Science and Technology; 2012; pp 295-348. 
(13) Bar, G.; Strum, G.; Gvishi, R.; Larina, N.; Lokshin, V.; Khodorkovsky, V.; Grinis, L.; Zaban, A.; Kiryuschev, I. Sol. Energy Mater. Sol. Cells 2009, 93, 2118-2124.

(14) Mihailovic, M.; Pataric, A.; Gulisija, Z.; Veljovic, D.; Janackovic, D. CI\&CEQ 2011, 17, $45-52$.

(15) Boccaccini, A. R.; Keim, S.; Ma, R.; Li, Y.; Zhitomirsky, I. J. R. Soc. Interface 2010, 7 , S581-S613.

(16) Mayr, H.; Ordung, M.; Ziegler, G. J. Mater. Sci. 2006, 41, 8138-8143.

(17) Oetzel, C.; Clasen, R. J. Mater. Sci. 2006, 41, 8130-8137.

(18) Anné, G.; Vanmeensel, K.; Vleugels, J.; Van der Biest, O. Key Engineering Materials 2006, $314,213-218$.

(19) Hamaker, H. C. Trans. Faraday Soc. 1940, 35, 279.

(20) Avgustinik, A. I.; Vigdergauz, V. S.; Zhuravlev, G. I. J. Appl. Chem. USSR 1962, 35, 21752180.

(21) Sarkar, P.; Nicholson, P. S. J. Am. Ceram. Soc. 1996, 79, 1987-2002.

(22) Biesheuvel, P. M.; Verweij, H. J. Am. Ceram. Soc. 1999, 82, 1451-1455.

(23) Kynch, G. J. Trans. Faraday Soc. 1952, 48, 166.

(24) González-Cuenca, M.; Biesheuvel, P. M.; Verweij, H. AIChE J. 2000, 46, 626-631.

(25) Ferrari, B.; Moreno, R. J. Eur. Ceram. Soc. 2010, 30, 1069-1078.

(26) Nold, A.; Assion, T.; Zeiner, J.; Clasen, R. Key Engineering Materials 2009, 412, 307-312.

(27) Falk, G. Key Engineering Materials 2012, 507, 155-162.

(28) Sullivan, K. T.; Kuntz, J. D.; Gash, A. E. Journal of Applied Physics 2012, 112, 024316. 
(29) Sullivan, K. T.; Worsley, M. A.; Kuntz, J. D.; Gash, A. E. Combust. Flame 2012, 159, 22102218.

(30) Riahifar, R.; Raissi, B.; Marzbanrad, E.; Zamani, C. J. Mater. Sci.: Mater. Electron. 2010, 22, 40-46.

(31) Pohl, H. A. Dielectrophoresis: The Behavior of Neutral Matter in Nonuniform Electric Fields; Cambridge University Press, 1978.

(32) Squires, T. M.; Bazant, M. Z. J. Fluid Mech. 2004, 509, 217-252.

(33) Russel, W. B.; Saville, D. A.; Schowalter, W. R. Colloidal Dispersions; Cambridge University Press: Cambridge England ; New York, 1989.

(34) Henry, D. C. P. Roy. Soc. Lond. A 1931, 133, 106-129.

(35) Bernal, J. D.; Mason, J. Nature 1960, 188, 910-911.

(36) Mooney, M. J. Colloid Sci. 1951, 6, 162-170.

(37) Deconinck, J. J. Appl. Electrochem. 1994, 24, 212-218. 